\title{
Gravity's whispers
}

\section{Proof positive.}

\section{Gregory Benford}

"The best is the enemy of the good," Sam said over my shoulder.

I whirled around, knowing the voice, smiling. "What —?"

He sauntered in, grinning in his lopsided way. "At 11 p.m. you're still working. Know your limits. The data can't get better when you're tired, y'know."

I threw down my pencil. "Right. Pursue the good. Let's get a beer."

At the Very Large Array, this meant a long drive back to Socorro. Our offices were there, but I liked spending time out among the big radio dishes, too. On the way back I rolled down the window to smell the tangy spring sagebrush and wondered whether Sam the Slow had finally decided to make a date with me, in his odd way. I'd been waiting half a year.

Then he said: "I was just passing by, thought I'd follow up on that puzzle I sent last week."

He had sent through a noise-dominated file. I had run one of my custom programs, got interested, and wasted a day pulling out a pattern. "You know me too well. I cracked it, yeah.” I gave him a smile he didn't notice. "Not a very interesting solution."

"You'd be surprised," Sam said, watching the desert slide by.

"It's you guys who surprised the world - the first gravity waves, wow."

"Yeah, decades of work on LIGO paid off."

Sam was also modest, a trait that gave him gal problems in the fanatic tech crowd more than once. Getting a gravitational wave to tweak a cavity, and detect that with interfering waves, had burned 20 years of his life. He shrugged. "We thought it was a signal from a rotating neutron star with a deformed crust. Say, you have that solution handy?"

I flipped open my laptop. "It's a string of numbers, turns out to be the zeroes of the Riemann zeta function."

"Uh huh. Which is -?"

"A famous function of complex argument. It analytically continues the sum of an infinite series."

"Sounds boring."

"Not so." At least he was looking at me now. "It's a big deal in analytic number theory, plenty of applications in physics, probability theory, Bose-Einstein condensates, spin waves -"

"Useful, good." Sam was usually sharp, focused, but now he gazed pensively at the stars.

"So how'd you get the detection?" It would help if I got him started about his
I blinked. "Can’t be. No natural system -"

"Exactly." Sam hooked an eyebrow at me.

"What? A tunable gravitational wave with a signal? That's im-"

“- possible, I know. Unless you can sling around neutron stars and make them sing in code."

Maybe, just maybe, this could be more important than at last getting Sam to date me. "Then ... you should know that it's not just a list of numbers. After 20 of the

Riemann zeros, there's something like a proof of the Riemann hypothesis."

He frowned. "Uh, so?"

"It's one of the greatest unsolved problems in mathematics. It says that any nontrivial zero has its real part exactly equal to $1 / 2$.'

He shook his head. "And that's the attention-catching opener to a SETI signal?"

"So you see, it can't be. Opening up with $\pi$ or $e$, prime numbers, the fine structure constant - that makes sense."

"Sense to the likes of us."

"So I must've made some mistake."

"No you didn't." Sam looked at me with a warm smile. "You're the only one I could run to with this analysis - the rest of 'em would laugh. You're good, really good."

I leaned over and kissed him. "Congratulations on the Nobel."

work - that is, his life. "You guys got rid of the noise from that road traffic and logging at the Louisiana site?"

"Yeah, took years. The signal we finally got had plenty of chirps and bursts in it, a bitch to clean up."

I grinned. Sam had worked for decades on LIGO, and now the milestone was here. "Now that you've got LIGO sensitive enough, there'll be plenty of signals. Supernovae in other galaxies, maybe rattling cosmic strings - "

"I want to understand this one. It's not a neutron star crust vibration, I think."

"Huh?" I was already tasting the beer in my mind.

"That decoding you did? That was our signal."
He kissed back, his eyes flickered, he grinned - but he didn't look happy. He grasped the steering wheel and peered ahead into the starlit darkness. In the high desert you can see stars above the headlights. I knew him enough to see that he was thinking about something that could whisper across the galaxies with gravitation, not using obvious means like radio or lasers. "Any mind that thinks the Riemann numbers are a calling card — and can throw around stars ..."

I got it. "Yeah. Know your limits. Maybe it's good, really good, that we can't possibly answer them."

Gregory Benford is a professor of physics at the University of California, Irvine, and the author of Timescape. 